\title{
Real-Time Imaging of the Medullary Circuitry Involved in the Generation of Spontaneous Muscle Sympathetic Nerve Activity in Awake Subjects
}

\author{
Vaughan G. Macefield ${ }^{1 *}$ and Luke A. Henderson ${ }^{2}$ \\ ${ }^{1}$ School of Medicine, University of Western Sydney, and Prince of Wales Medical Research Institute, \\ Sydney, Australia \\ ${ }^{2}$ Department of Anatomy and Histology, University of Sydney, Sydney, Australia
}

\begin{abstract}
To understand the central neural processes involved in blood pressure regulation we recorded muscle sympathetic nerve activity (MSNA) via a tungsten microelectrode in the common peroneal nerve while performing functional magnetic resonance imaging (fMRI) of the brainstem at 3T. Blood oxygen level dependent (BOLD) changes in signal intensity were measured over $4 \mathrm{~s}$ every $8 \mathrm{~s}$ (200) volumes; MSNA was recorded during the previous $4 \mathrm{~s}$ epoch, which takes into account peripheral conduction delays along unmyelinated axons and neurovascular coupling delays. Analysis of temporal coupling between BOLD signal intensity and nerve signal intensity revealed sites in which the two signals covaried, but only in the medulla. Because scans were conducted in a caudorostral direction, we could constrain the analysis to the medulla by only examining the first $1 \mathrm{~s}$ of the fMRI and nerve signals. Increases in MSNA were associated with robust bilateral increases in signal intensity in the dorsolateral region of the medulla that corresponds to the human equivalent of the rostal ventrolateral medulla (RVLM). Reciprocal decreases in signal intensity occurred in the regions of the nucleus tractus solitarius (NTS) and caudal ventrolateral medulla (CVLM). Group analysis also revealed increases in signal intensity in the caudal pressor area (CPA), medullary raphé (MR), and dorsal motor nucleus of the vagus (DMX). We have shown for the first time that this combined approach of recording sympathetic neural activity and fMRI provides real-time imaging of the neural processes responsible for the generation of sympathetic nerve activity in awake human subjects. Hum Brain Mapp 31:539549, 2010. @ 2009 Wiley-Liss, Inc.
\end{abstract}

Key words: baroreflex; medullary nuclei; sympathetic nervous system; RVLM; CVLM; NTS

Contract grant sponsor: National Health and Medical Research Council of Australia; Contract grant number: 350889.

*Correspondence to: Prof. Vaughan G. Macefield, School of Medicine, University of Western Sydney, Locked Bag 1797, Penrith South DC, NSW 1797, Australia. E-mail: v.macefield@uws.edu.au Received for publication 19 November 2008; Revised 20 July 2009; Accepted 20 July 2009

DOI: $10.1002 / \mathrm{hbm} .20885$

Published online 23 September 2009 in Wiley InterScience (www. interscience.wiley.com).

\section{INTRODUCTION}

The automatic control of diverse organ systems provided by the sympathetic and parasympathetic divisions of the autonomic nervous system is vital for the homeostatic adjustments essential in life, and there are many pathophysiological conditions in which disorders in autonomic control have been identified. While much has been learnt about the brainstem and hypothalamic circuitry largely responsible for this control from studies in anaesthetized experimental animals, studies in awake human subjects have mostly been limited to noninvasive studies on the effector organs of the autonomic nervous system 
(by measuring changes in heart rate, blood pressure, skin blood flow, sweat release, etc) or invasive studies in which the activity of sympathetic postganglionic neurons is recorded via percutaneous intraneural microelectrodes (microneurography). However, little is known about the central nervous control of the autonomic nervous system in awake human subjects.

A primary determinant of resting blood pressure is the level of sympathetically-mediated vasoconstriction within skeletal muscle. Microneurographic studies have shown that muscle sympathetic nerve activity (MSNA) at rest, which occurs as pulse-synchronous bursts and is measured as burst frequency (bursts per minute) or burst incidence (bursts per 100 heart beats) is consistent in a given individual from day to day, year to year [Fagius and Wallin, 1993]. Some subjects have high levels of resting MSNA and some have low levels; identical twins have similar levels, whereas the burst incidence in fraternal twins differs widely [Wallin et al., 1993]. While there is a good inverse relationship between cardiac output and the level of resting MSNA across a group of healthy subjects [Charkoudian et al., 2005], there is a poor relationship between resting MSNA and resting blood pressure; accordingly, one cannot predict the level of MSNA in a given healthy individual by recording blood pressure (or heart rate) alone [Joyner et al., 2008]. However, it is clear that in a given subject bursts of MSNA occur when diastolic pressure is low-and hence baroreceptor input is low-during the spontaneous fluctuations in arterial pressure that occur at rest [Sundlöf and Wallin, 1978]. The purpose of the present study was to record spontaneous fluctuations in MSNA, brought about by the spontaneous fluctuations in blood pressure, and to use this pattern of neural activity-coupled with functional magnetic resonance imaging (fMRI) of the brainstem-to functionally identify sites within the brainstem that are responsible for the spontaneous fluctuations in resting MSNA.

It is known from animal work that the nucleus tractus solitarius (NTS) is the primary receiving area of inputs from the arterial baroreceptors; the NTS sends excitatory projections to the caudal ventrolateral medulla (CVLM), which then exerts tonic inhibitory (GABAergic) control of the rostral ventrolateral medulla (RVLM) - the primary output nucleus responsible for the sympathetic vasoconstrictor control of arterial pressure [for review see Dampney et al., 2003a,b; Guyenet, 2006]. Although this baroreflex circuitry is well defined in experimental animals, it is not known if a similar set of brainstem structures mediate spontaneous baroreflex activity in awake humans. By recording spontaneous MSNA concurrently with fMRI of the brainstem we aim to determine if these three medullary nuclei are also involved in the baroreflex in human subjects. Based on animal work we will test the hypothesis (i) that the occurrence of bursts of MSNA is associated with an increase in signal intensity in RVLM and a reciprocal decrease in activity of the NTS and CVLM. In addition to these three medullary regions, it has recently been shown in animals that a pressor region immediately caudal to the CVLM, the caudal pressor area (CPA), exerts a tonic facilitation on neurons within RVLM [Horiuchi et al., 2004]. Given this, we shall test the hypothesis (ii) that activity within the CPA covaries with that in RVLM. By recording spontaneous MSNA concurrently with blood oxygen level dependent (BOLD) signal intensity changes within the human brainstem, we aim to identify the medullary nuclei involved in the baroreflex, and hence the generation of spontaneous bursts of MSNA, in human subjects. This combination of direct recording of sympathetic nerve activity and functional magnetic resonance imaging (fMRI) is unique, and has not been attempted previously, yet promises to provide us with important information on the central processes responsible for autonomic control in awake human subjects.

\section{METHODS}

Ten experiments were performed on eight healthy subjects (six male, two female), aged 18-29, under approval of the Human Research Ethics Committee of the University of New South Wales. All experiments were conducted at the Symbion Clinical Research Imaging Centre at Prince of Wales Medical Research Institute (Achieva 3T, Philips Medical Systems, The Netherlands). Subjects lay supine on an MRI bed in a laboratory. A knee was supported on a foam block and the common peroneal nerve located at the fibular head by electrical stimulation through a surface probe (3-10 mA, $0.2 \mathrm{~ms}, 1 \mathrm{~Hz}$; Stimulus Isolator, ADInstruments, Sydney, Australia). An insulated tungsten microelectrode (FHC, Maine, USA) was inserted percutaneously into the nerve and manually guided into a muscle fascicle of the nerve while delivering weak electrical stimuli to evoke muscle twitches $(0.01-1 \mathrm{~mA}, 0.2 \mathrm{~ms}, 1 \mathrm{~Hz})$. A nearby subdermal microelectrode, with $1 \mathrm{~mm}$ insulation removed, served as the reference electrode and a surface $\mathrm{AgCl}$ electrode on the leg as the ground electrode. Once a muscle fascicle had been entered neural activity was amplified (gain $10^{4}$, bandpass $0.3-5.0 \mathrm{kHz}$ ) using a lownoise, electrically isolated, headstage (NeuroAmpEX, ADInstruments, Australia). The innervation territory of the muscle fascicle was identified by tapping over the muscle belly or relevant tendon, and the position of the microelectrode tip manually adjusted until spontaneous bursts of muscle sympathetic nerve activity (MSNA) were identified. Because the amplifier headstage is encased in stainless steel, and contains no iron components, it was intrinsically safe to operate within the high-field MRI environment. Pilot experiments confirmed that the amplifier did not block during the application of the high-frequency radio frequency $(R F)$ pulses during the scanning sequences, and that the frequencies of interest within the nerve recording lay within the RF band and therefore could not be retained by RF filters. Neural activity was acquired, RMS-processed (200 ms) and analyzed on computer (Chart 5, 
PowerLab 25T; ADInstruments, Australia). A high-pass digital filter at $300 \mathrm{~Hz}$ was applied to the recorded signal to remove artifacts picked up by the cable from the headstage to the amplifier.

The subject's head was enclosed in an eight channel SENSE head coil and stabilized with foam pads to minimize head movement. Headphones were provided to minimize noise and to allow communication with the subject. A continuous series of 200 gradient echo echo-planar images, sensitive to blood oxygen level dependent (BOLD) contrast and encompassing the entire brainstem (extending from the top of the thalamus to the top of the cervical spinal cord) were collected over a $27-\mathrm{min}$ period (46 axial slices, $\mathrm{TR}=8 \mathrm{~s}, \mathrm{TE}=40 \mathrm{~ms}$, flip angle $=90^{\circ}$, raw voxel size $=1.5 \mathrm{~mm}^{3}$ ). All 46 axial slices were collected sequentially from caudal to rostral during the first $4 \mathrm{~s}$ of the 8 -s TR, such that the first parts of the scan were limited to the medulla whereas the later parts of the scan included the pons and thalamus. Sections of the MSNA recordings that contained the scanning artifacts were removed and the remaining data, which contained unaffected MSNA signals, were spliced together. Mean voltage levels of the RMS nerve signal, reflecting fluctuations in MSNA (or the underlying noise in the absence of MSNA bursts), were measured automatically every second during each interscan period.

Functional images were motion corrected and a brainstem template created from the functional image set of one subject. All subjects' images were then normalized to this template, intensity normalized to remove slow signal intensity drifts and smoothed using a 3-mm FWHM Gaussian filter. Both motion and global signal were included as nuisance variables. Changes in fMRI signal intensity were assessed during the subsequent 4-s period to take into account the $\sim 5 \mathrm{~s}$ neurovascular coupling delay and the $\sim 1 \mathrm{~s}$ required for conduction of the sympathetic bursts from the brainstem to the peripheral recording site (see Discussion). As we were interested in defining medullary regions responsible for baroreflex regulation, correlations between MSNA during the first second of the subsequent 4-s recording period and BOLD signal changes in medullary slices, which were collected during the first second of the brainstem image collection period, were made. Medullary regions correlated to MSNA were then determined for all 7 trials and overlaid onto an individual subject's functional image set (fixed effects, $P<0.05$, corrected for multiple comparisons, six-direction movement parameters included as nuisance variables, medulla only mask). In addition, signal changes correlated to MSNA were assessed in individual subjects (uncorrected $P>0.005$ ). For significantly correlated clusters in individual subjects, the mean signal intensity during the $20 \mathrm{vol}$. corresponding to the highest MSNA was compared to the mean signal intensity during the 20 vol. corresponding to the lowest MSNA (two sample $t$-test, $P<0.05)$. For those subjects in whom significant differences were observed, the percentage change in MSNA and BOLD signal intensity were plotted against each other, and a linear correlation analysis performed. Finally, changes in signal intensity of the significant clusters were plotted over time.

\section{RESULTS}

A typical recording from our laboratory of spontaneous muscle sympathetic nerve activity (MSNA) at rest is shown in Figure 1. It can be seen that MSNA occurs as waves of bursts: each burst exhibited strong cardiac rhythmicity, and each wave of bursts occurred during spontaneous falls in blood pressure. It can also be seen that heart rate increased in parallel, and that muscle sympathetic nerve activity (as well as heart rate) was inhibited as blood pressure was increased by the sympathetically-mediated vasoconstriction. Concurrent recordings of MSNA and fMRI of the brainstem were achieved in eight experiments. In one of the remaining experiments, the recording site was lost before the sequence was completed; in another the subject had too little MSNA at rest to provide sufficient data for analysis. A sample of the neural data obtained from one subject is shown in Figure 2. Despite the large scanning artifacts, stable neural recordings were obtained, although the background electrical noise levels were higher in the scanner. It can be seen in Figure 2 that spontaneous bursts of MSNA were observed in some, but not all, of the 4-s intervals between scans. Given that the apparent burst incidence during scanning was no different to that observed during the resting period, recorded prior to or following the scanning sequence, we can reasonably conclude that the scanning per se did not change a subject's resting level of MSNA.

Significant correlations between brainstem signal intensity and MSNA, obtained from eight experiments, were found when the cumulative burst amplitude of the MSNA signal, calculated over the entire 4-s inter-scan period, was used as the input model. Analysis of temporal coupling between BOLD signal intensity and nerve signal intensity revealed sites in which the two signals covaried, but these sites were limited to the medulla. By examining the first $1 \mathrm{~s}$ of the fMRI and nerve signals we could constrain our analyses to the medulla. Group average data are shown in Figure 3: sagital sections (medial to lateral) are shown in the left panel and axial sections (rostral to caudal) in the right panel. Increases in MSNA were associated with robust bilateral increases in signal intensity in the dorsolateral region of the medulla that corresponds to the human equivalent of the rostal ventrolateral medulla (RVLM; see Discussion). There was also a unilateral (left) increase in activity in the area that we believe corresponds to the caudal pressor area (CPA). Reciprocal decreases in signal intensity occurred in the dorsomedial region of the nucleus tractus solitarius (NTS) and in the caudal ventrolateral medulla (CVLM), though (on average) the latter appeared to be limited to the right side. As illustrated in Figure 4 (upper panel) group analysis also revealed increases in 

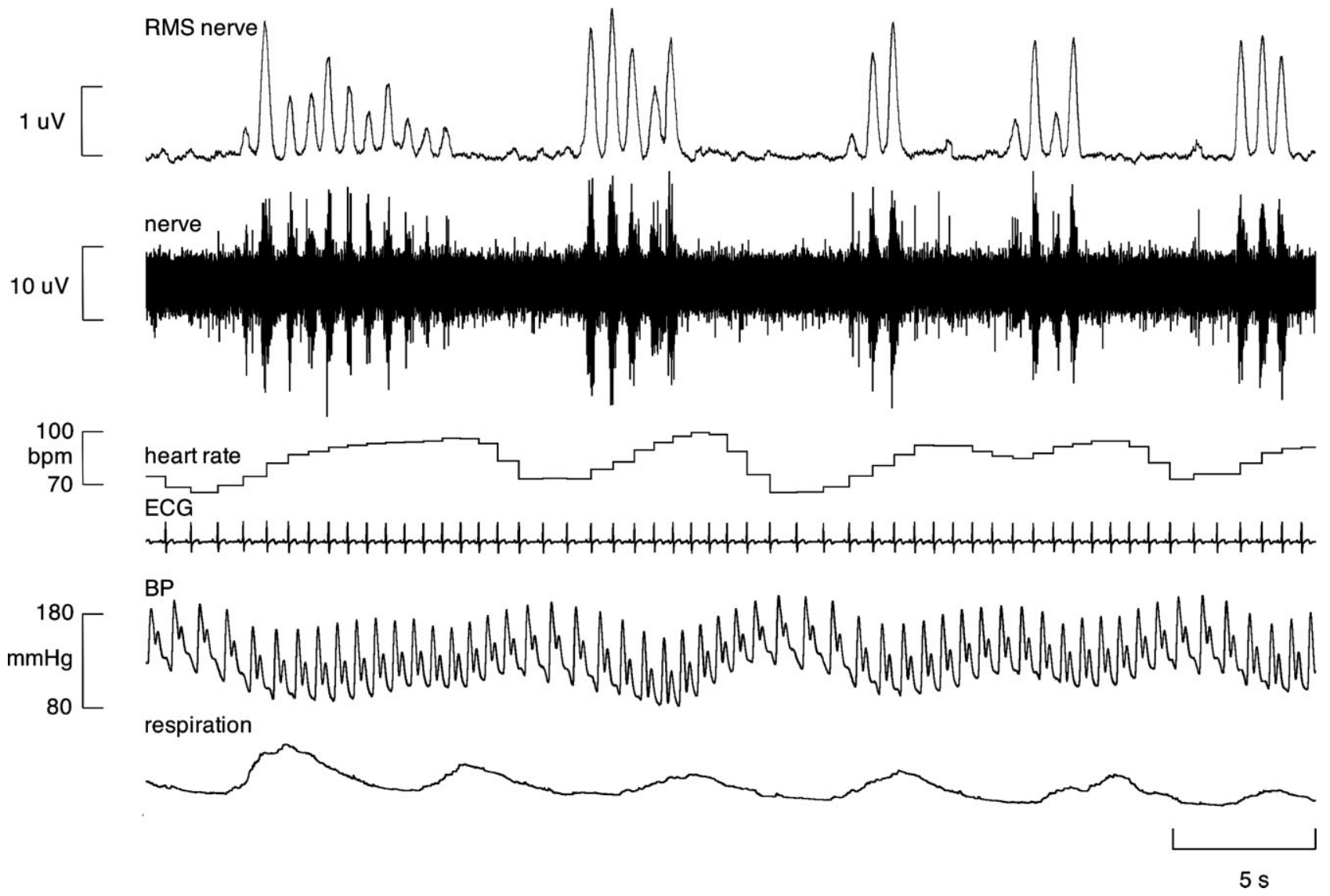

Figure I.

Laboratory recording of spontaneous muscle sympathetic nerve activity (MSNA), ECG, and continuous blood pressure (recorded noninvasively) in a healthy subject at rest.

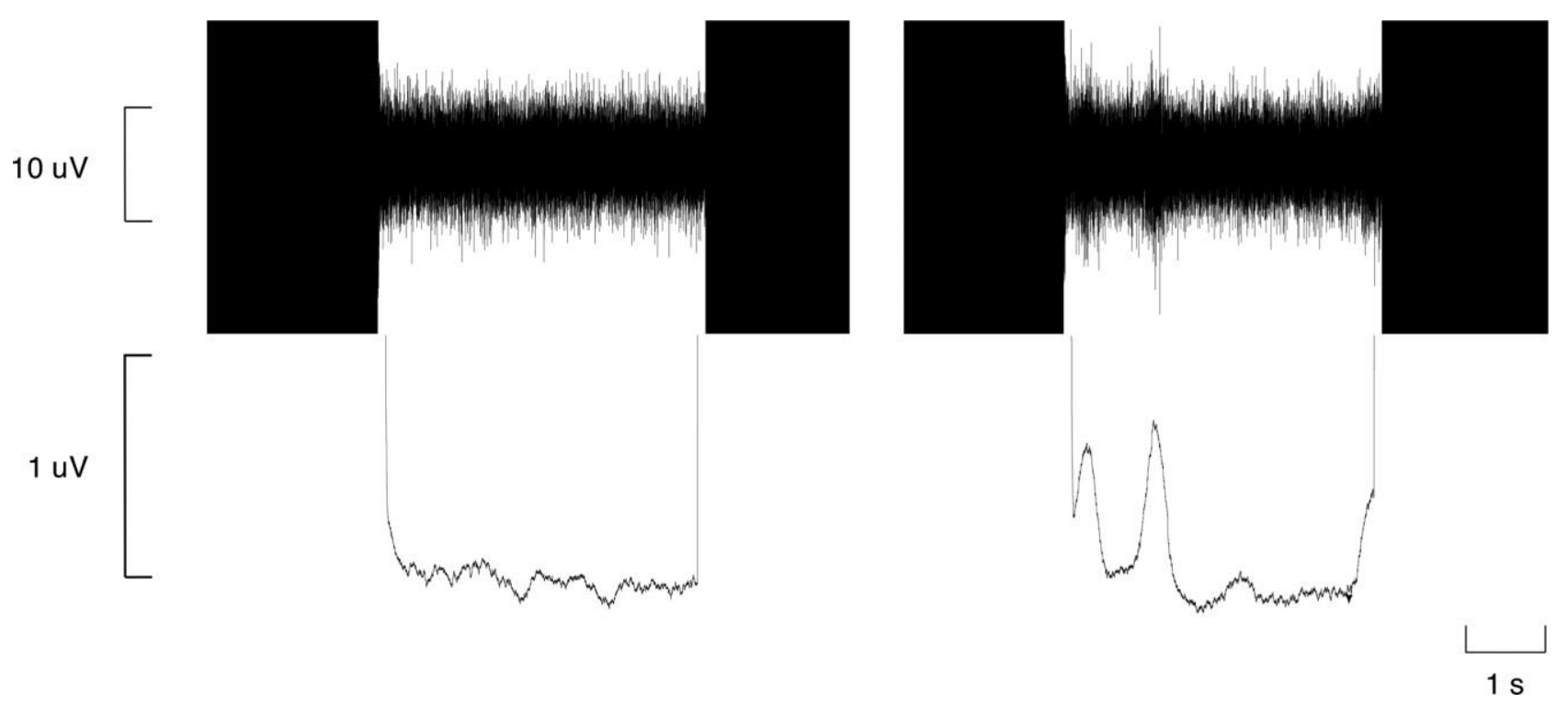

Figure 2.

Spontaneous MSNA recorded during scanning. Filtered data are shown in the top trace, RMSprocessed data in the lower trace. The dense areas are scanning artifacts. 

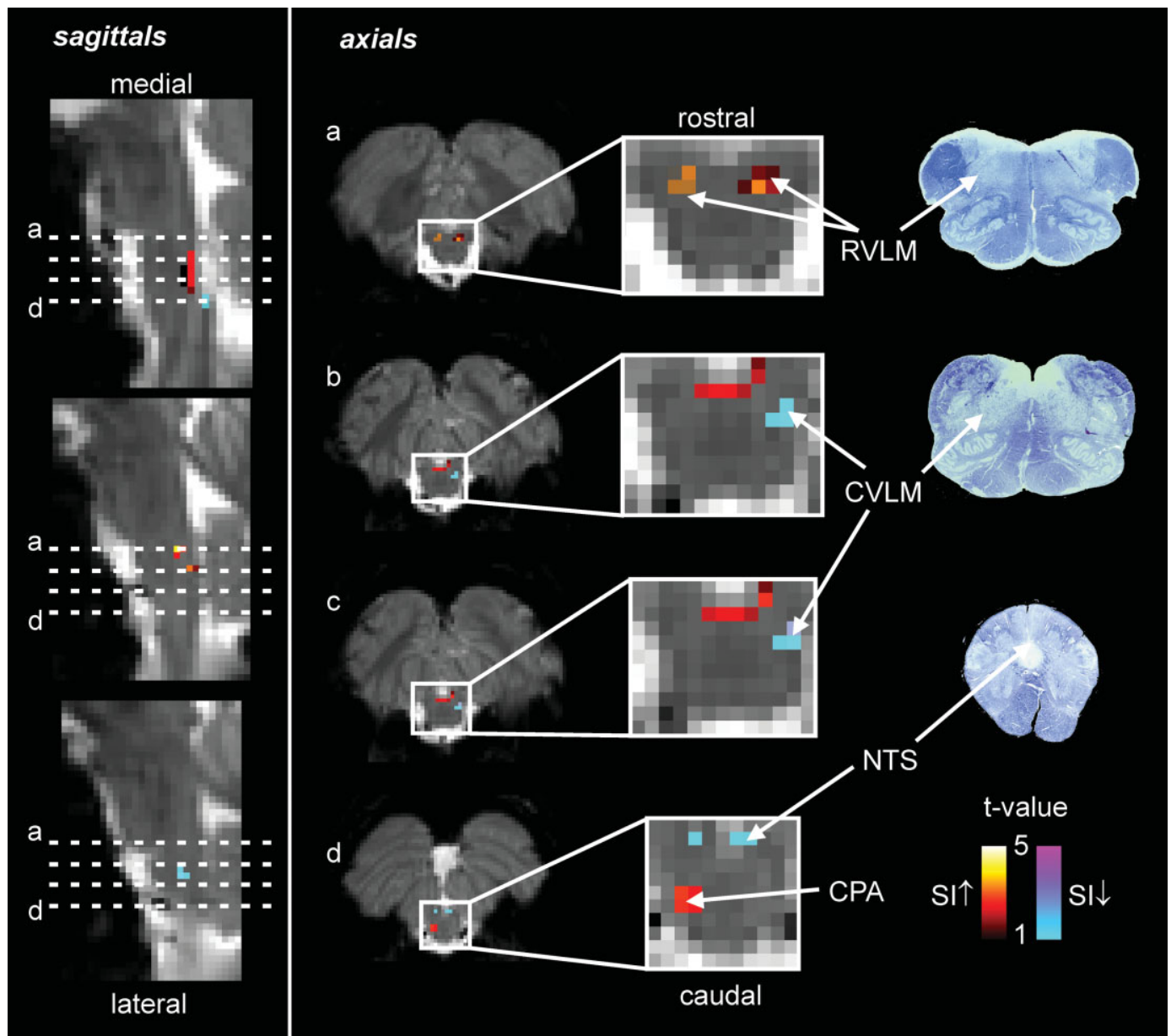

Figure 3.

Signal intensity changes positively (warm color scale) and negatively (cool color scale) correlated with MSNA in the resting state overlaid onto an individual subjects $\mathrm{FMRI}$ image set. Group data from nine experiments. Each $\mathrm{fMRI}$ voxel is $1.5 \times 1.5 \times$ $1.5 \mathrm{~mm}$. Equivalent myelin-stained histological sections are

signal intensity in the medullary raphe and in the region that includes the dorsal motor nucleus of the vagus (DMX).

Given the inverse relationship between spontaneous MSNA and diastolic pressure at rest (see Fig. 1), we reasoned that when MSNA is high-and the input from the baroreceptors low-NTS and CVLM activity would be low and RVLM activity would be high. The lower panel of Fig. 4 shows individual datasets from six subjects after splitting the data into periods in which MSNA was high (the top $20 \%$ of signal amplitudes) and low (the bottom $20 \%$ ). It is apparent that signal intensity in RVLM was higher for those periods in which MSNA was high, and significantly lower in those periods in which MSNA was low. Although the scatter was large, plotting these two clusters of data revealed significant positive linear correlations between MSNA and RVLM signal intensity. shown on the right. RVLM, rostral ventrolateral medulla; CVLM, caudal ventrolateral medulla; NTS, nucleus tractus solitarius (solitary tract nucleus); CPA, caudal pressor area; SI, signal intensity. Sagital sections (medial to lateral) are shown in the left panel, axial sections (rostral to caudal) in the right.

Similar data are shown for the caudal ventrolateral medulla (CVLM) for five subjects in Figure 5. Again, when the data were separated into periods in which MSNA was high or low, there were significant differences in signal intensity within the CVLM. However, the relationship between CVLM activity and MSNA was the inverse of that between RVLM activity and MSNA: when MSNA was high CVLM activity was low, and when MSNA was low CVLM activity was high. Although this failed to reach statistical significance for Subject 5, it is clear that the same trend was observed.

Time-series data, illustrating covariation of MSNA and signal intensity in RVLM and CVLM, are shown for two subjects in Figure 6. In both subjects fluctutations in signal intensity within RVLM largely occurred in parallel with the fluctuations in MSNA. Conversely, fluctuations in signal intensity in CVLM essentially mirrored those occurring 


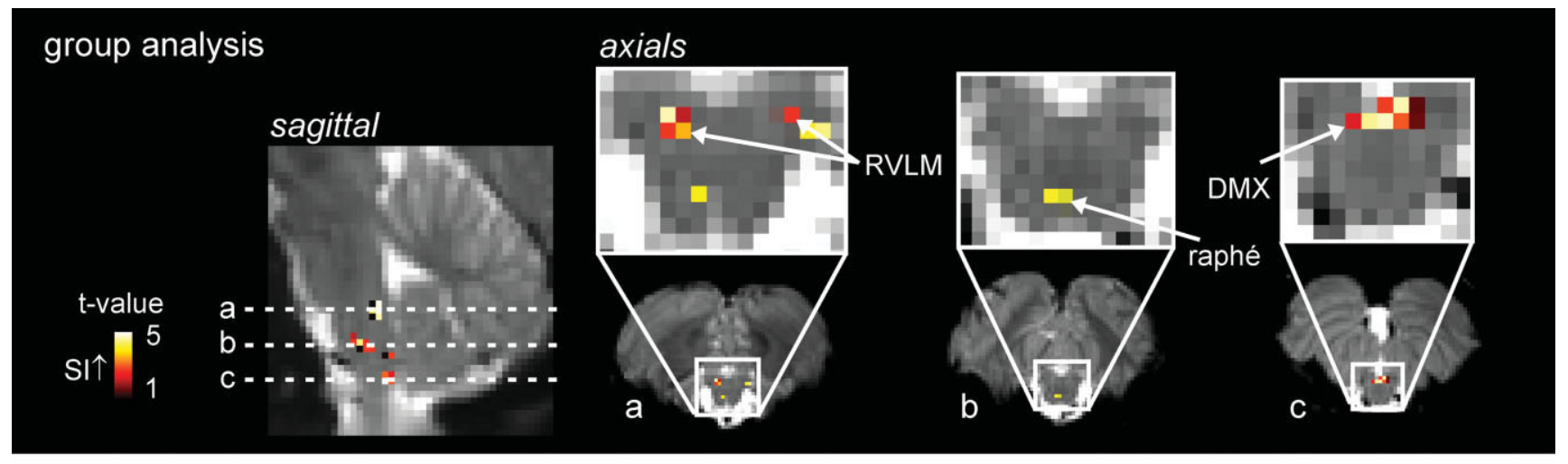

individual subjects - RVLM
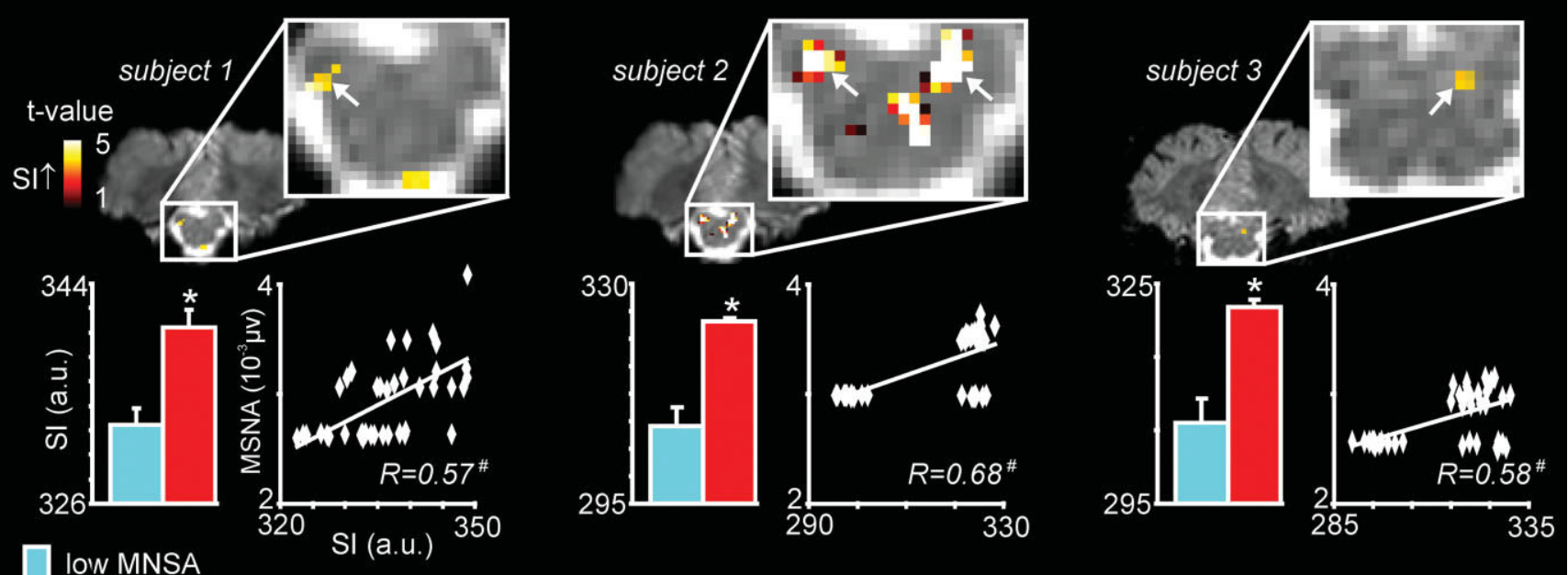

$\square$ high MNSA
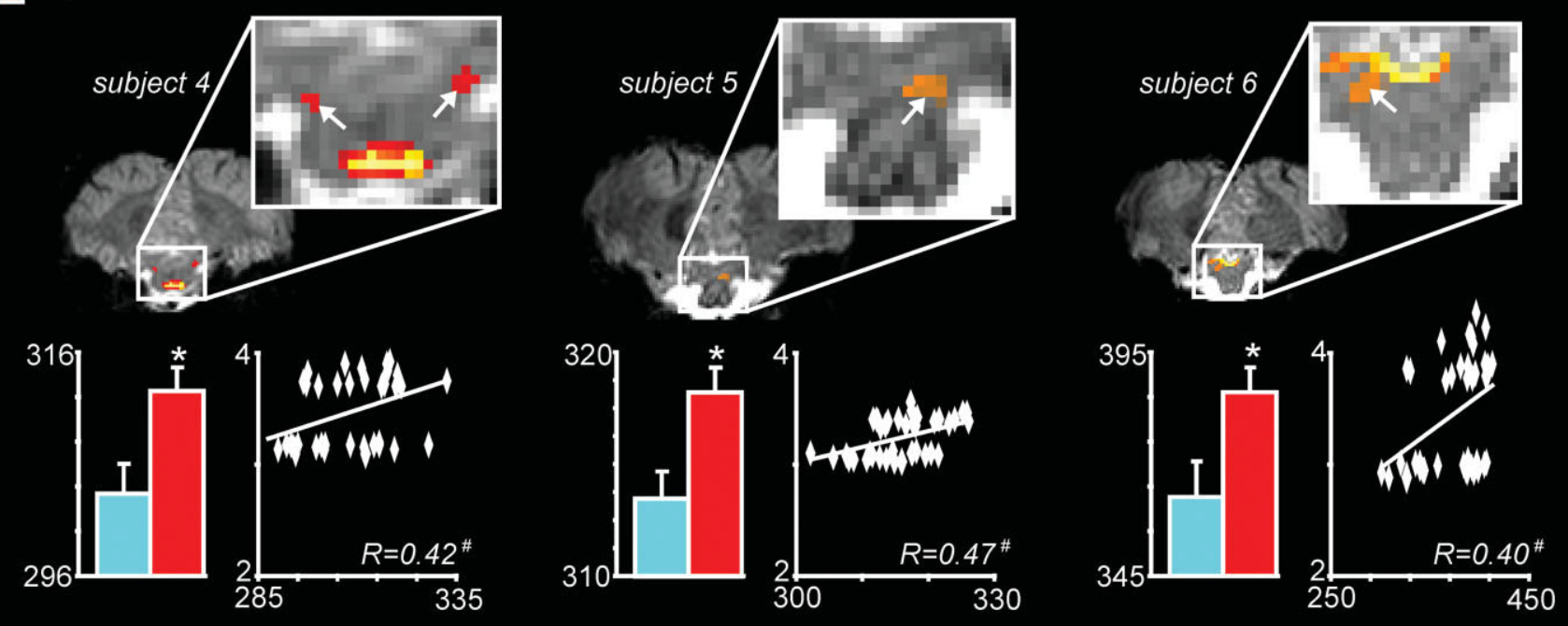

Figure 4.

The top panel shows the group analysis showing increased signal and negatively (cool color scale) correlated to MSNA, overlaid intensity in rostral ventrolateral medulla (RVLM), medullary raphe and the dorsal motor nucleus of the vagus (DMX). The bottom panel shows for six subjects signal intensity in RVLM after dividing the data into periods associated with high muscle sympathetic nerve activity (MSNA) (red) and low MSNA (blue). Regions in which signal intensity is positively (hot color scale) onto functional MR images are shown in the inset of each panel. Data from all subjects show that the increases in signal intensity within RVLM were significantly higher when MSNA was high. SI, signal intensity. $*$ represents a significant difference in signal intensity during high and low MSNA conditions. 


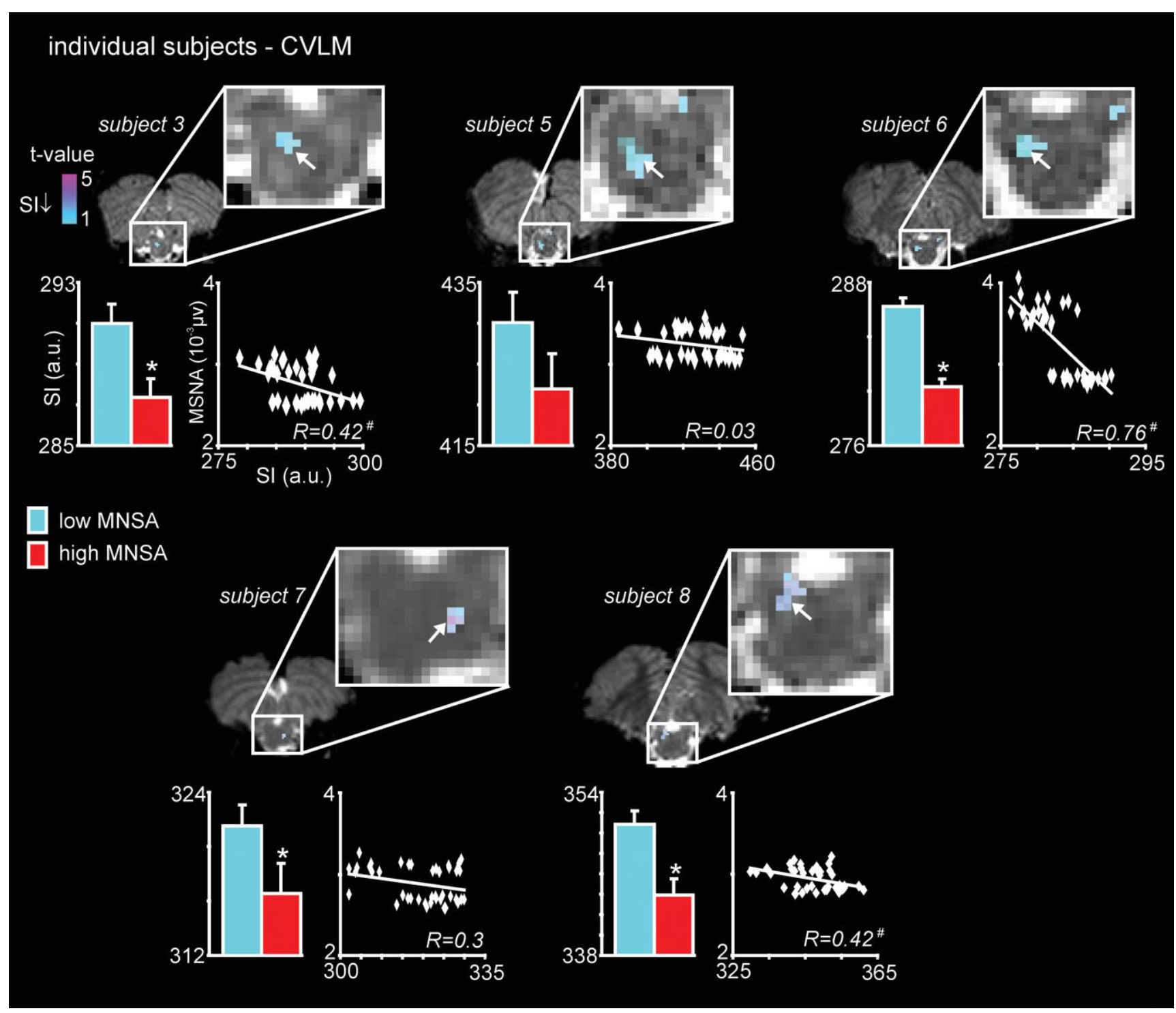

Figure 5.

Differences in signal intensity within caudal ventrolateral medulla (CVLM) after dividing the data into periods associated with high muscle sympathetic nerve activity (MSNA) (red) and low MSNA (blue). Regions in which signal intensity is positively (hot color scale) and negatively (cool color scale) correlated to MSNA, overlaid onto functional MR images are shown in the inset of each panel. Data from five subjects. Signal intensity in CVLM was high when MSNA was low and low when MSNA was high. $\mathrm{SI}$, signal intensity. ${ }^{*}$ represents a significant difference in signal intensity during high and low MSNA conditions. in the nerve signal. Data from all subjects are shown graphically in Figure 7.

\section{DISCUSSION}

By recording spontaneous MSNA concurrently with BOLD signal in the brainstem we aimed to identify the medullary baroreflex circuitry in human subjects and hence identify regions responsible for the generation of spontaneous muscle vasoconstrictor nerve activity. On the basis of animal work we predicted that a decrease in activity of the NTS would occur during spontaneous falls in arterial pressure, resulting in decreases in activity in CVLM and hence increases in RVLM. Our data demonstrate the feasibility of undertaking intraneural recordings from awake human subjects in a high-field MRI environment, and confirm the predicted changes in signal intensity in NTS, CVLM, and RVLM. 


\section{subject 1}
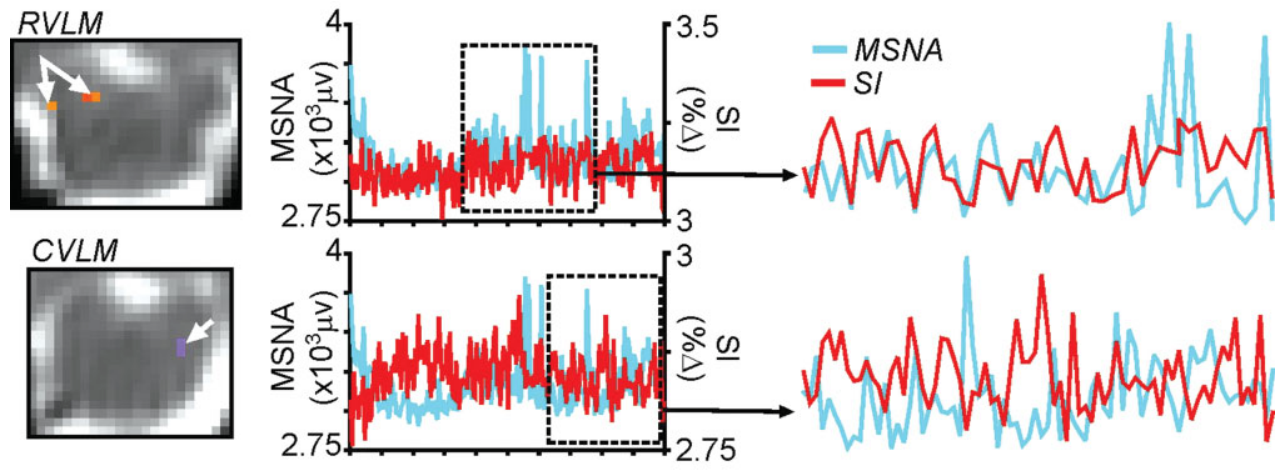

subject 2
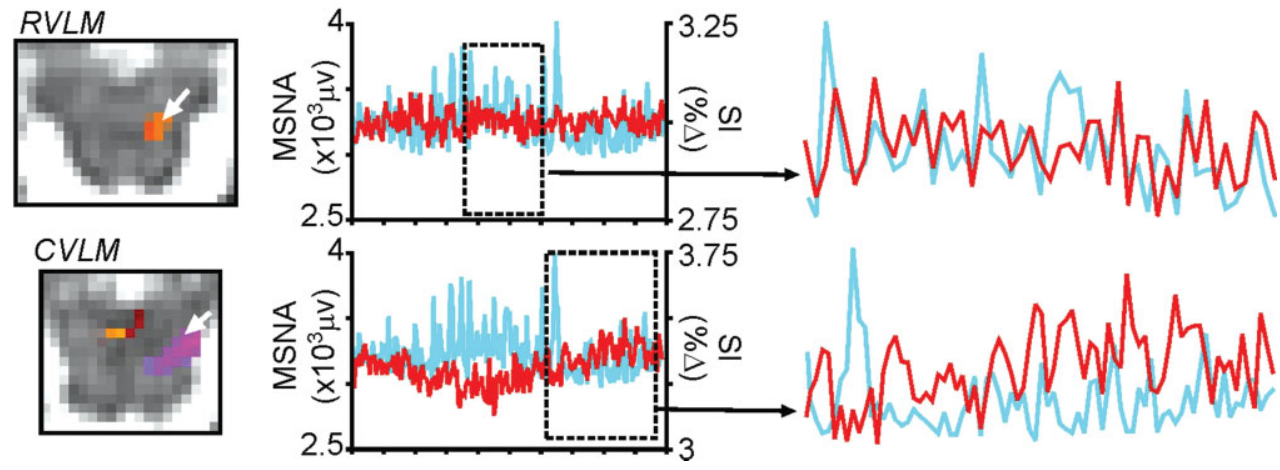

Figure 6.

Covariation of signal intensity within rostral ventrolateral medulla (RVLM) and muscle sympathetic nerve activity (MSNA) and caudal ventrolateral medulla (CVLM) and MSNA in two subjects. Regions in which signal intensity is positively (hot color scale) and negatively (cool color scale) correlated to MSNA, overlaid onto functional $M R$ images are shown to the left and the

\section{Identification of the Medullary Nuclei Responsible for the Baroreflex in Experimental Animals}

The fundamental circuitry responsible for the baroreflex has been examined over many decades in anaesthetized experimental animals. Early work had shown that excitation within circumscribed regions of the RVLM could induce increases in blood pressure and activation of vasoconstrictor neurons supplying skeletal muscle, while activation within other areas could induce increases in activity in cutaneous sympathetic neurons yet no increase in blood pressure [Dampney and McAllen, 1988; McAllen et al., 1995]. Moreover, electrolytic destruction of neurons within RVLM was shown to cause a precipitous fall in blood pressure, arguing that ongoing activity within RVLM is essential for resting vasomotor tone [Dampney and Moon, 1980]. It is known from intracellular recordings from RVLM neurons that they exhibit an irregular tonic changes in signal intensity within these regions to the right. In both subjects, the fluctuations in MSNA and signal intensity in RVLM occurred essentially in parallel, while fluctuations in MSNA and signal intensity in CVLM essentially mirrored each other. Expanded sections are shown in the far right.

firing that decreases with increases in blood pressure and increases with decreases in blood pressure; it is also known that this tonic activity is modified by excitatory and inhibitory inputs, but that in the absence of such inputs this tonic activity continues [Dampney et al., $2003 a, b]$. The current understanding of the baroreflex circuitry-defined primarily in anaesthetized animal preparations-is that primary afferent axons from the baroreceptors project to the caudal region of the NTS, where they synapse onto second-order neurons, which in turn send excitatory (glutamatergic) projections onto GABAregic neurons within the region of the CVLM. These CVLM GABAergic neurons synapse directly onto excitatory neurons within the RVLM and serve to inhibit the spontaneous activity of RVLM premotor sympathetic neurons. Nucleus ambiguus and the dorsal motor nucleus of the vagus also receive glutamatergic projections from NTS [Wang et al., 2001], activating vagal cardiac efferents and 


\section{RVLM}

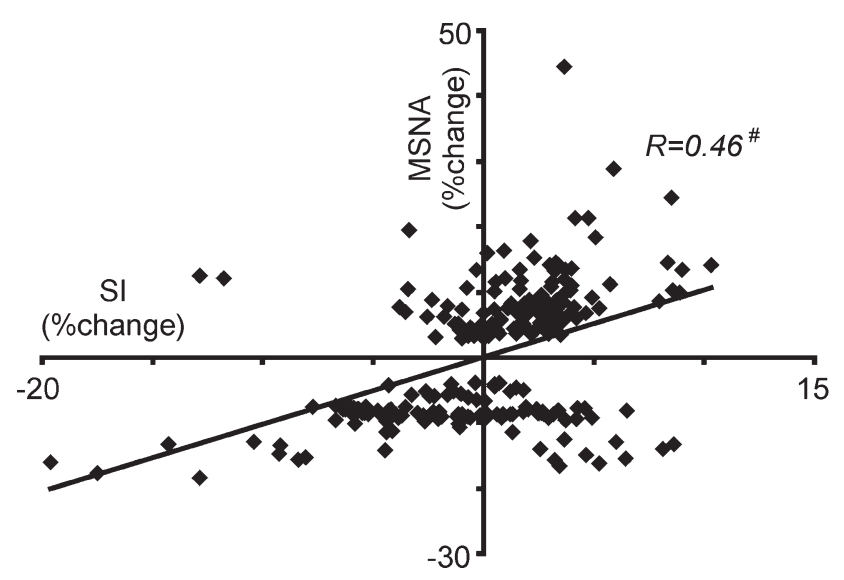

CVLM

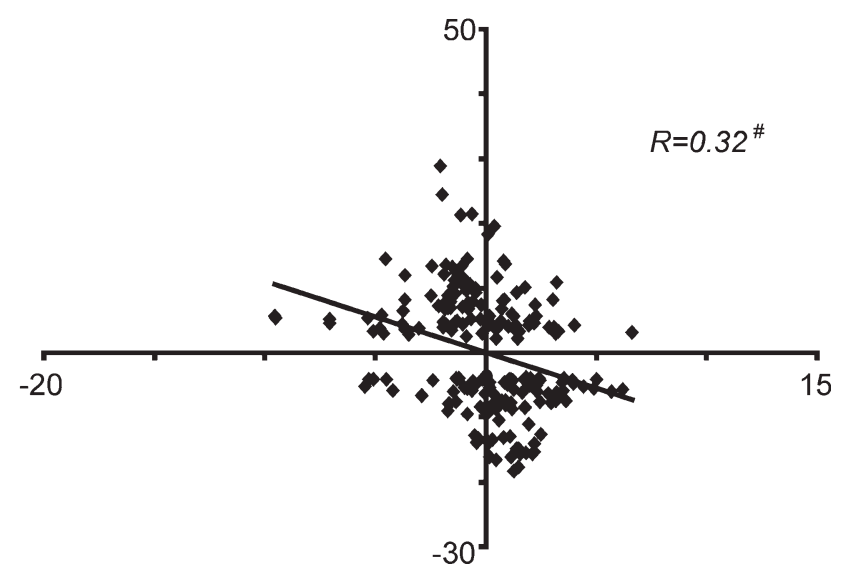

Figure 7.

Covariation of muscle sympathetic nerve activity (MSNA) and signal intensity within rostral ventrolateral medulla (RVLM) and caudal ventrolateral medulla (CVLM). MSNA was positively correlated to RVLM signal intensity and negatively correlated to CVLM signal intensity.

slowing the heart. In addition to these components of the basic baroreflex arc, it is known that other brainstem regions, such as the caudal pressor area (CPA), located in the most caudal part of the ventrolateral medulla, can also influence baroreflex activity by altering the activity in these baroreflex medullary nuclei [Dampney et al., 2003a,b]. Studies in conscious animals, using c-fos expression as a marker of neuronal activation, have confirmed the operation of the NTS-CVLM-RVLM serial pathway during manouevres that increase or decrease arterial pressure [Dampney and Horiuchi, 2003; Minson et al., 1997].

\section{Identification of Medullary Cardiovascular Nuclei in Human Subjects}

While fMRI in the anaesthetized cat has revealed changes in signal intensity within these same regions of the medulla [Henderson et al., 2004a,b], relatively few studies have applied fMRI to investigate the role of the brainstem in human cardiovascular control. One of the first studies to address this used the Valsalva manoeuvre, a forced expiratory effort that causes a sustained increase in muscle sympathetic nerve activity and blood pressure. Significant changes in BOLD signal intensity occurred in many areas of the brain, including the dorsal pons and medulla [Harper et al., 2000; Henderson et al., 2002]. We recently showed that a maximal inspiratory breath-hold caused significant changes in BOLD signal intensity in three discrete regions of the medulla: robust increases in signal intensity occurred in the region of the RVLM and decreases in signal intensity occurred within the regions of the CVLM and NTS [Macefield et al., 2006]. These signal intensity changes were expected, given that the increase in
MSNA during this manouevre is believed to be due to unloading of the low-pressure baroreceptors [Macefield, 1998; Macefield et al., 2006]. In humans, the RVLM and CVLM are displaced dorsally by the large inferior olivary nuclei [Allen et al., 1998]. The functional and anatomical localization of the human RVLM is illustrated in Figure 8: the left panel shows a bilateral increase in RVLM signal intensity during a maximal inspiratory breath-hold [data reanalyzed from Macefield et al., 2006]; the right panel shows the anatomical identification of the human RVLM, based on histochemical identification [Allen et al., 1998].

In the present study, we examined spontaneous fluctuations in muscle sympathetic outflow and documented the same changes within the medulla as we had previously identified during the sustained increases in MSNA produced by a maximal inspiratory breath hold. Although investigations into brainstem sites involved in producing sustained changes in sympathetic drive are important, the changes in brainstem activity evoked by these challenges can be influenced by higher brain centers that regulate functions such as arousal and attention. By measuring spontaneous fluctuations in MSNA we are circumventing these issues and can therefore measure a more accurate representation of moment-to-moment baroreflex activity. This is important, as it may be the case that in some autonomic diseases the performance of a particular maneuver may result in similar brain activity changes and similar changes in blood pressure and MSNA even though the underlying baroreceptor reflex arc may be significantly altered. The use of concurrent recordings of spontaneous MSNA and brainstem fMRI signal changes creates an opportunity to investigate the activity of human brainstem nuclei in baseline autonomic states in subjects with and without a range of autonomic conditions. 


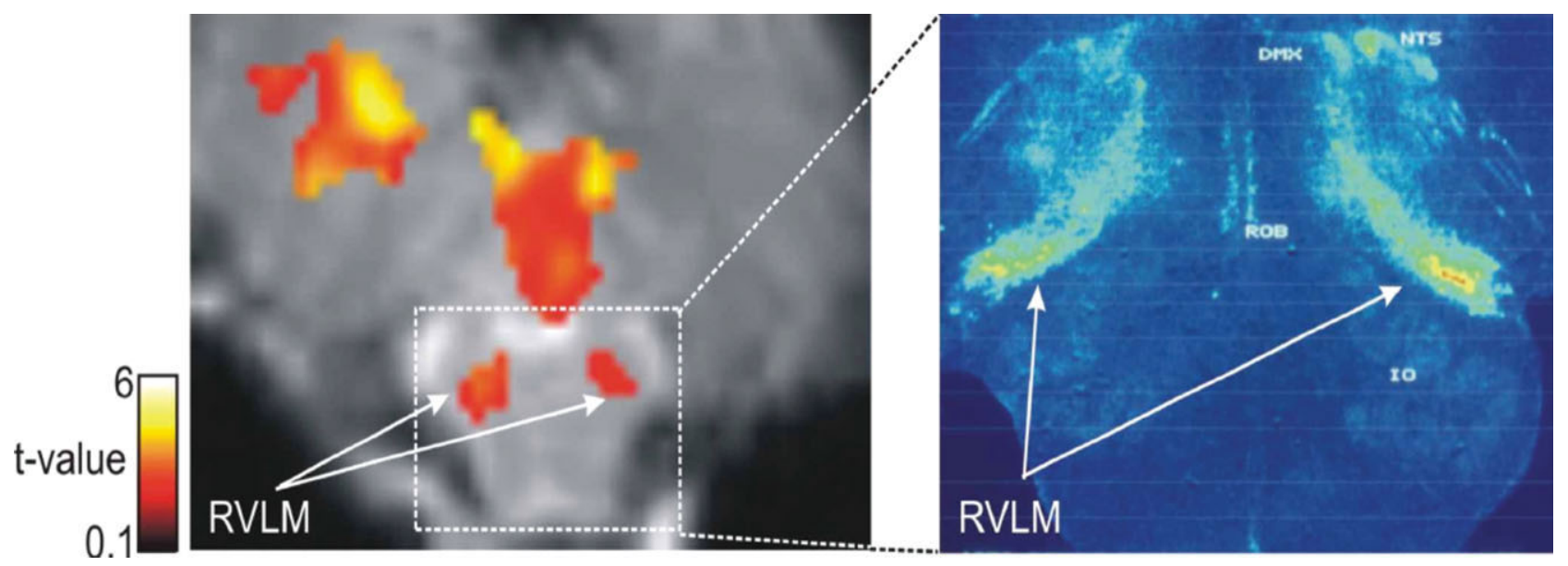

Figure 8.

Functional and anatomical localization of the human RVLM. The left panel shows a bilateral increase in RVLM signal intensity during a maximal inspiratory breath-hold [data reanalyzed from Macefield et al., 2006]; the right panel shows the anatomical identification of the human RVLM, based on the presence of Angiotensin II receptors [figure reproduced with permission from Allen et al., 1998].

In addition to the increase in signal intensity in RVLM and, reciprocal decreases in intensity in NTS and CVLM, we observed increases in signal intensity in the caudal pressor area (CPA), dorsal motor nucleus of the vagus (DMX), and the medullary raphé (MR). While the parallel increase in CPA activity can be explained by the known excitatory projections from CPA to RVLM [Dampney et al., 2003a,b], it is more difficult to explain the increase in DMX: given that heart rate increases during spontaneous increases in MSNA, we would expect a decrease in signal intensity in DMX-further studies in which cardiodeceleration is induced will be required to specifically address this issue. We were also surprised by the parallel increase in signal intensity in the medullary raphé. Given that the raphé is believed to be primarily responsible for cutaneous vasoconstrictor activity [Tanaka et al., 2002] and McAllen et al. [2006] have shown increases in BOLD signal intensity in this region during whole-body cooling in human subjects, we would not expect there to be covariation with spontaneous muscle vasoconstrictor activity.

\section{Methodological Considerations}

We used an ON-OFF scanning protocol so that we could record MSNA during the 4-s period between each 4-s scan. This interval was chosen to take advantage of the temporal delays inherent in BOLD imaging. It is known that the microvascular responses to an increase in neuronal activity lag by some $5 \mathrm{~s}$ [Logothetis et al., 2001], and that we need to allow $\sim 1 \mathrm{~s}$ for a muscle vasoconstrictor volley to travel from the brainstem to the peripheral recording site at the knee [Fagius and Wallin, 1980]. Accordingly, we reasoned that changes in BOLD signal intensity would reflect changes in neural activity associated with emission of sympathetic volleys recorded in the previous 4-s epoch.

There is convincing evidence that increases in BOLD signal intensity reflect the underlying electrical events within the brain [Logothetis et al., 2001], though whether this reflects neuronal firing or synaptic events is a matter of contention [Viswanathan and Freeman, 2007]. Moreover, there is controversy as to what a decrease in signal intensity reflects, with different authors referring to these decreases as "inhibitions" or, more correctly, "deactivations," though recent data indicates that both activation and deactivation patterns of BOLD signal intensity correlate well with neuronal glucose oxidation in glutamatergic neurons [Kida and Hyder, 2006]. On the basis our results, we would argue that a decrease in signal intensity, such as observed in NTS and CVLM, reflects a decrease in neuronal firing rather than synaptic events per se, given that the synaptic events within RVLM are related to the active release of the inhibitory neurotransmitter GABA. Finally, given that we could document spontaneous fluctuations in brainstem BOLD signal intensity that were time-locked to changes in muscle sympathetic nerve activity (and by inference, changes in blood pressure), we believe that much of the "baseline noise" in brainstem BOLD signal is simply a reflection of these underlying physiological processes.

\section{CONCLUSIONS}

We have demonstrated that it is possible to record muscle sympathetic nerve activity while undertaking fMRI of the brain. Moreover, we have demonstrated the operation of the fundamental medullary circuitry involved in the 
baroreflex and the generation of spontaneous muscle vasconstrictor drive.

\section{ACKNOWLEDGMENTS}

The authors are grateful to Kirsten Moffat for her assistance in running the scans and Paul Nash for his assistance with the experiments.

\section{REFERENCES}

Allen AM, Moeller I, Jenkins TA, Zhuo J, Aldred GP, Chai SY, Mendelsohn FA (1998): Angiotensin receptors in the nervous system. Brain Res Bull 47:17-28.

Charkoudian N, Joyner MJ, Johnson CP, Eisenach JH, Dietz NM, Wallin BG (2005): Balance between cardiac output and sympathetic nerve activity in resting humans: Role in arterial pressure regulation. J Physiol 568:315-321.

Dampney RA, Moon EA (1980): Role of ventrolateral medulla in vasomotor response to cerebral ischemia. Am J Physiol 239: H349-H358.

Dampney RA, McAllen RM (1988): Differential control of sympathetic fibres supplying hindlimb skin and muscle by subretrofacial neurones in the cat. J Physiol 395:41-56.

Dampney RA, Horiuchi J (2003): Functional organisation of central cardiovascular pathways: Studies using c-fos gene expression. Prog Neurobiol 71:359-384.

Dampney RA, Horiuchi J, Tagawa T, Fontes MA, Potts PD, Polson JW (2003a): Medullary and supramedullary mechanisms regulating sympathetic vasomotor tone. Acta Physiol Scand 177: 209-218.

Dampney RA, Polson JW, Potts PD, Hirooka Y, Horiuchi J (2003b): Functional organization of brain pathways subserving the baroreceptor reflex: Studies in conscious animals using immediate early gene expression. Cell Mol Neurobiol 23:597-616.

Fagius J, Wallin BG (1980): Sympathetic reflex latencies and conduction velocities in normal man. J Neurol Sci 47:433-348.

Fagius J, Wallin BG (1993): Long-term variability and reproducibility of resting human muscle nerve sympathetic activity at rest, as reassessed after a decade. Clin Auton Res 3:201-205.

Guyenet PG (2006): The sympathetic control of blood pressure. Nat Rev Neurosci 7:335-346.

Harper RM, Bandler R, Spriggs D, Alger JR (2000): Lateralized and widespread brain activation during transient blood pressure elevation revealed by magnetic resonance imaging. J Comp Neurol 417:195-204.

Henderson LA, Macey PM, Macey KE, Frysinger RC, Woo MA, Harper RK, Alger JR, Yan-Go FL, Harper RM (2002): Brain responses associated with the Valsalva maneuver revealed by functional magnetic resonance imaging. J Neurophysiol 88:3477-3486.
Henderson LA, Macey PM, Richard CA, Runquist ML, Harper RM (2004a): Functional magnetic resonance imaging during hypotension in the developing animal. J Appl Physiol 97:2248-2257.

Henderson LA, Richard CA, Macey PM, Runquist ML, Yu PL, Galons JP, Harper RM (2004b): Functional magnetic resonance signal changes in neural structures to baroreceptor reflex activation. J Appl Physiol 96:693-703.

Horiuchi J, Killinger S, Dampney RA (2004): Contribution to sympathetic vasomotor tone of tonic glutamatergic inputs to neurons in the RVLM. Am J Physiol 287:R1335-R1343.

Joyner MJ, Charkoudian N, Wallin BG (2008): A sympathetic view of the sympathetic nervous system and human blood pressure regulation. Exp Physiol 93:715-724.

Kida I, Hyder F (2006): Physiology of functional magnetic resonance imaging: Energetics and function. Methods Mol Med 124:175-195.

Logothetis NK, Pauls J, Augath M, Trinath T, Oeltermann A (2001): Neurophysiological investigation of the basis of the fMRI signal. Nature 412:150-157.

Macefield VG (1998): Sustained activation of muscle sympathetic outflow during lung inflation depends on a high intrathoracic pressure. J Autonom Nerv Syst 68:135-139.

Macefield VG, Gandevia SC, Henderson LA (2006): Neural sites involved in the sustained increase in muscle sympathetic nerve activity induced by inspiratory-capacity apnea-A fMRI study. J Appl Physiol 100:266-273.

McAllen RM, May CN, Shafton AD (1995): Functional anatomy of sympathetic premotor cell groups in the medulla. Clin Exp Hypertens 17:209-221.

McAllen RM, Farrell M, Johnson JM, Trevaks D, Cole L, McKinley MJ, Jackson G, Denton DA, Egan GF (2006): Human medullary responses to cooling and rewarming the skin: A functional MRI study. Proc Natl Acad Sci USA 103:809-813.

Minson JB, Llewellyn-Smith IJ, Arnolda LF, Pilowsky PM, Chalmers JP (1997): C-fos expression in central neurons mediating the arterial baroreceptor reflex. Clin Exp Hypertens 19:631-643.

Sundlöf G, Wallin BG (1978): Human muscle nerve sympathetic activity at rest. Relationship to blood pressure and age. J Physiol 274:621-637.

Tanaka M, Nagashima K, McAllen RM, Kanosue K (2002): Role of the medullary raphé in thermoregulatory vasomotor control in rats. J Physiol 540:657-664.

Viswanathan A, Freeman RD (2007): Neurometabolic coupling in cerebral cortex reflects synaptic more than spiking activity. Nat Neurosci 10:1308-1312.

Wallin BG, Kunimoto MM, Sellgren J (1993): Possible genetic influence on the strength of human muscle nerve sympathetic activity at rest. Hypertens 22:282-284.

Wang J, Irnaten M, Neff RA, Venkatesan P, Evans C, Loewy AD, Mettenleiter TC, Mendelowitz D (2001): Synaptic and neurotransmitter activation of cardiac vagal neurons in the nucleus ambiguus. Ann N Y Acad Sci 940:237-246. 